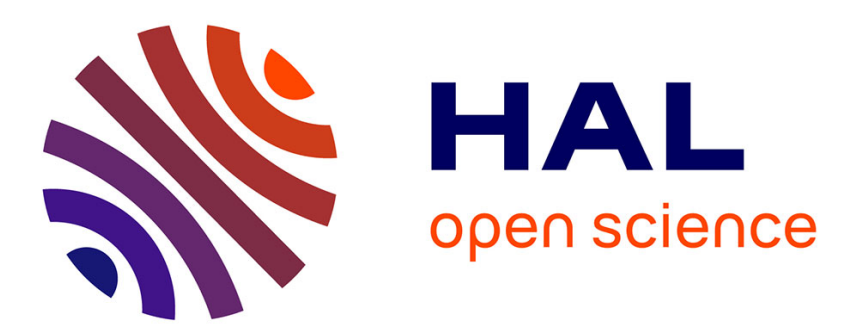

\title{
Étude théorique et expérimentale de la génération harmonique acoustique dans le molybdate de plomb
}

R. Torguet, E. Bridoux

\section{To cite this version:}

R. Torguet, E. Bridoux. Étude théorique et expérimentale de la génération harmonique acoustique dans le molybdate de plomb. Revue de Physique Appliquée, 1972, 7 (4), pp.291-302. 10.1051/rphysap:0197200704029100 . jpa-00243631

\section{HAL Id: jpa-00243631 https://hal.science/jpa-00243631}

Submitted on 1 Jan 1972

HAL is a multi-disciplinary open access archive for the deposit and dissemination of scientific research documents, whether they are published or not. The documents may come from teaching and research institutions in France or abroad, or from public or private research centers.
L'archive ouverte pluridisciplinaire HAL, est destinée au dépôt et à la diffusion de documents scientifiques de niveau recherche, publiés ou non, émanant des établissements d'enseignement et de recherche français ou étrangers, des laboratoires publics ou privés. 


\title{
ÉTUDE THÉORIQUE ET EXPÉRIMENTALE DE LA GÉNÉRATION HARMONIQUE ACOUSTIQUE DANS LE MOLYBDATE DE PLOMB
}

\author{
R. TORGUET \\ Laboratoire Central de Recherches Thomson-CSF, 91, Orsay \\ E. BRIDOUX \\ Laboratoire d'Ultrasons, Centre Univèrsitaire, 59, Valenciennes \\ (Reçu le 5 juin 1972, révisé le 28 juillet 1972)
}

\begin{abstract}
Résumé. - En utilisant la théorie non linéaire de l'élasticité, nous avons calculé les amplitudes des harmoniques qui prennent naissance dans un cristal de molybdate de plomb lors de la propagation d'ondes ultrasonores d'amplitude finie. Grâce aux propriétés acousto-optiques remarquables du molybdate de plomb, nous avons pu visualiser directement les effets non linéaires. Un montage optique superhétérodyne a permis de détecter des puissances de l'ordre du nanowatt et de mesurer avec précision la constante de couplage $\Gamma=3+C_{333} / C_{33}$.
\end{abstract}

\begin{abstract}
Using non-linear elasticity theory, calculations of the amplitudes of harmonics generated in a lead molybdate crystal by finite amplitude ultrasonic waves have been made. Due to excellent acousto-optical properties of lead molybdate, we have directly vizualized non-linear effects. An optical heterodyne method has allowed detection of nanowatt acoustic power levels and the accurate determination of the coupling constant $\Gamma=3+C_{333} / C_{33}$.
\end{abstract}

I. Introduction. - L'interaction de la lumière avec les ondes acoustiques de haute fréquence est un phénomène important par ses nombreuses applications dans tous les systèmes de communication optique et de traitement de l'information utilisant des lasers [1]-[5].

Parallèlement au développement de ces techniques, des études systématiques ont été faites pour sélectionner les matériaux ayant les meilleures performances requises (rendement acousto-optique élevé, faible perte acoustique, stabilité en température, etc.) [6]-[9]. Le molybdate de plomb $\mathrm{PbMoO}_{4}$ est actuellement le matériau le plus utilisé dans les applications optiques, [10] à cause de son facteur de mérite acoustooptique élevé, et de ses faibles pertes acoustiques et optiques.

De plus, il n'est pas soluble dans l'eau, ce qui explique son avantage par rapport à l'acide alpha-iodique $\left(\alpha-\mathrm{HIO}_{3}\right)$ qui a un facteur de mérite comparable [11].

L'utilisation de transducteurs de niobate de lithium $\mathrm{LiNbO}_{3}$ à fort coefficient de couplage électromécanique permet d'obtenir des puissances acoustiques élevées. Avec une puissance hyperfréquence d'entrée de 2,5 W sur le déflecteur utilisé dans cette étude, la puissance acoustique est égale à $1 \mathrm{~W}$ et pratiquement $100 \%$ de la lumière incidente est déviée. Pour des ondes acoustiques de densité de puissance de l'ordre de $10 \mathrm{~W} / \mathrm{cm}^{2}$, la loi de Hooke n'est plus valable [12]. Les anharmonicités du réseau cristallin déforment les ondes acoustiques d'amplitude finie au cours de leur propagation et il y a transfert d'une partie de l'énergie de l'onde fondamentale à ses harmoniques [13]-[16].

Ces effets non linéaires peuvent être expliqués mathématiquement en introduisant les constantes élastiques du troisième ordre dans l'équation $d u$ mouvement [17]. Nous établirons d'abord dans le cas $\mathrm{du} \mathrm{PbMoO}_{4}$ les équations couplées entre les amplitudes des différents harmoniques. En utilisant les techniques du calcul numérique, nous avons obtenu la variation amplitudes en fonction de la distance parcourue dans le cristal.

Nous avons utilisé une sonde optique pour détecter les différentes harmoniques. Il suffit de faire varier l'angle d'incidence d'un faisceau laser sur les plans d'onde acoustique et de translater le cristal par rapport au laser et au photodétecteur qui restent fixes pour étudier la variation de la puissance transportée par chaque harmonique au cours de leur propagation dans le cristal [18]. Cette méthode a l'avantage d'éviter la mesure de la perte de conversion électromécanique d'un système détecteur à chaque fréquence, la puissance lumineuse diffractée étant indépendante de la fréquence acoustique.

Nous avons cherché à mettre au point une technique de mesure permettant de mesurer avec précision la constante de couplage qui caractérise les non-linéarités acoustiques du $\mathrm{PbMoO}_{4}$. L'appareillage réalisé au cours de ce travail nous a permis de détecter des puissances acoustiques de l'ordre du nanowatt et de visualiser les ondes harmoniques. 
II. Etude théorique de la génération harmonique d'ondes acoustiques. - A. EQUATION NON LINÉAIRE DU MOUVEMENT. - Les équations générales de propagation des ondes acoustiques d'amplitude finie dans les solides ont été écrites par Thurston [19]. Dans ce travail, nous utiliserons les coordonnées lagrangiennes. La position instantanée de chaque atome du cristal est exprimée en fonction de sa position initiale dans le cristal en équilibre. Toute grandeur physique dépendant du mouvement des atomes du cristal est fonction $\mathrm{du}$ temps et de la position initiale des atomes. Si les $\left(a_{j}\right)$ sont les coordonnées des atomes à l'état d'équilibre, les positions instantanées $\left(x_{j}\right)$ des atomes dans le cristal déformé sont fonction des $\left(a_{j}\right)$ et du temps. Il en est de même pour le vecteur déplacement $u$ défini par la relation :

$u_{i}\left(a_{j}, t\right)=x_{i}\left(a_{j}, t\right)-a_{i} \quad i=1,2,3, j=1,2,3$.

La déformation en chaque point du cristal s'exprime à l'aide du tenseur des déformations dont les composantes $\eta_{i j}$ sont définies de la façon suivante :

$$
\eta_{i j}=\frac{1}{2}\left[\frac{\partial u_{i}}{\partial a_{j}}+\frac{\partial u_{j}}{\partial a_{i}}+\frac{\partial u_{k}}{\partial a_{i}} \cdot \frac{\partial u_{k}}{\partial a_{j}}\right] .
$$

Thurston [19] introduit une tension thermodynamique $t_{i j}$ définie par:

$$
t_{i j}=-\frac{\partial U}{\partial \eta_{i j}}
$$

où $U$ est l'énergie libre isentropique du cristal. Pour des cristaux non piézo-électriques soumis à des déformations élastiques, l'énergie libre peut être développée par rapport aux variables élastiques $\eta_{i j}$. Si ce développement est limité au troisième ordre, nous obtenons :

$$
U=-\frac{1}{2} C_{i j k l} \eta_{i j} \eta_{k l}-\frac{1}{6} C_{i j k l m n} \eta_{i j} \eta_{k l} \eta_{m n}
$$

où $C_{i j k l}, C_{i j k l m n}$ sont respectivement les constantes élastiques du second et du troisième ordre, et la tension thermodynamique peut s'écrire :

$$
t_{i j}=C_{i j k l} \eta_{k l}+\frac{1}{2} C_{i j k l m n} \eta_{k l} \eta_{m n} .
$$

En remplaçant la tension thermodynamique par l'expression précédente dans l'équation générale de propagation obtenue par Thurston [19] :

$$
\rho_{0} \frac{\partial^{2} u_{i}}{\partial t^{2}}=\frac{\partial}{\partial a_{m}}\left(\frac{\partial x_{i}}{\partial a_{k}}\right) t_{k m}
$$

où $\rho_{0}$ est la masse volumique dans l'état non déformé, nous obtenons l'équation non linéaire du mouvement [20] :

$$
\rho_{0} \frac{\partial^{2} u_{i}}{\partial t^{2}}=\frac{\partial^{2} u_{k}}{\partial a_{j} \partial a_{m}}\left[C_{i j k m}+\frac{\partial u_{p}}{\partial a_{q}} M_{i j k m p q}\right]
$$

avec

$$
\begin{aligned}
M_{i j k m p q}=C_{i j k m p q}+\delta_{k p} C_{i j m q} & + \\
& +\delta_{i k} C_{j m p q}+\delta_{i p} C_{j k m q} .
\end{aligned}
$$

Cette équation du mouvement se simplifie dans le cas de la propagation suivant des axes principaux qui correspondent à des modes acoustiques purs. Pour les ondes acoustiques longitudinales se propageant suivant la direction $(0,0,1)$ du molybdate de plomb, cristal tétragonal appartenant à la classe $4 / \mathrm{m}$, nous obtenons à partir des constantes élastiques non nulles $\mathrm{du}$ second et du troisième ordre déterminées par Brugger [21] pour toutes les classes cristallographiques :

$\rho_{0} \frac{\partial^{2} u_{3}}{\partial t^{2}}=C_{33} \frac{\partial^{2} u_{3}}{\partial a_{3}^{2}}+\left(3 C_{33}+C_{333}\right) \frac{\partial^{2} u_{3}}{\partial a_{3}^{2}} \cdot \frac{\partial u_{3}}{\partial a_{3}}$

où :

$$
\frac{\partial^{2} u_{3}}{\partial a_{3}^{2}}-\frac{1}{v^{2}} \frac{\partial^{2} u_{3}}{\partial t^{2}}=-\frac{\Gamma}{2} \frac{\partial}{\partial a_{3}}\left(\frac{\partial u_{3}}{\partial a_{3}}\right)^{2}
$$

avec

$$
\begin{aligned}
& v^{2}=\frac{C_{33}}{\rho_{0}} \quad \text { (étant la vitesse des ondes acoustiques) } \\
& \Gamma=3+\frac{C_{333}}{C_{33}} \quad \text { (constante de couplage) }
\end{aligned}
$$

Dans le premier membre de l'éq. (9), nous retrouvons l'équation linéaire du mouvement obtenue en limitant au second ordre le développement de l'énergie libre du cristal.

B. EquATIONS COUPLÉEs. - Nous nous limiterons dans cette étude à la génération des quatre premiers harmoniques et nous pouvons donc écrire le déplacement $u_{3}$ sous la forme :

$$
u_{3}=\sum_{n=1}^{4}\left[P_{n}\left(a_{3}\right) \cdot C(n)+Q_{n}\left(a_{3}\right) \cdot S(n)\right]
$$

avec

$$
\begin{aligned}
& C(n)=\cos n\left(K a_{3}-\Omega t\right) \\
& S(n)=\sin n\left(K a_{3}-\Omega t\right) .
\end{aligned}
$$

Les amplitudes $P_{n}\left(a_{3}\right)$ et $Q_{n}\left(a_{3}\right)$ ne peuvent pas être considérées comme constantes à cause de l'existence de termes non linéaires dans le second membre de l'éq. (9). Nous supposons qu'elles sont des fonctions lentement variables de la coordonnée $a_{3}$ de sorte que :

$P_{n}\left(a_{3}\right) \gg \frac{\Gamma_{1}}{n K} \frac{\mathrm{d} P_{n}\left(a_{3}\right)}{\mathrm{d} a_{3}} \gg\left(\frac{1}{n K}\right)^{2} \cdot \frac{\mathrm{d}^{2} P_{n}\left(a_{3}\right)}{\mathrm{d} a_{3}} \gg \cdots$.

En remplaçant $u_{3}$ par l'expression (10) dans l'équation $\mathrm{du}$ mouvement (9) et en tenant compte des hypothèses précédentes, nous obtenons l'expression de l'équation de propagation : 


$$
\begin{aligned}
\sum_{n=1}^{4} n\left[\frac{\mathrm{d} Q_{n}}{\mathrm{~d} a_{3}}\right. & \left.C(n)-\frac{\mathrm{d} P_{n}}{\mathrm{~d} a_{3}} S(n)\right]=-\frac{\Gamma K^{2}}{4} \sum_{n=1}^{4}\left\{n^{3}\left[\left(P_{n}^{2}-Q_{n}^{2}\right) \cdot S(2 n)-2 P_{n} Q_{n} \cdot C(2 n)\right]+\right. \\
& +\sum_{p<n} n p\left[(n+p)\left(P_{n} P_{p}-Q_{n} Q_{p}\right) \cdot S(n+p)-(n+p)\left(P_{n} Q_{n}+P_{p} Q_{n}\right)\right. \\
& \left.\left.\times C(n+p)-(n-p)\left(P_{n} P_{p}+Q_{n} Q_{p}\right) \cdot S(n-p)+(n-p)\left(P_{p} Q_{n}-P_{n} Q_{p}\right) \cdot C(n-p)\right]\right\} .
\end{aligned}
$$

En identifiant les termes de même fréquence et de même phase, nous obtenons les équations couplées suivantes :

$$
\begin{aligned}
& \frac{\mathrm{d} Q_{1}}{\mathrm{~d} a_{3}}=-\frac{\Gamma K^{2}}{2}\left[\left(P_{1} Q_{2}-P_{2} Q_{1}\right)+3\left(P_{2} Q_{3}-P_{3} Q_{2}\right)+6\left(P_{3} Q_{4}-P_{4} Q_{3}\right)\right] \\
& \frac{\mathrm{d} P_{1}}{\mathrm{~d} a_{3}}=\frac{\Gamma K^{2}}{2}\left[-\left(P_{1} P_{2}+Q_{1} Q_{2}\right)-3\left(P_{2} P_{3}+Q_{2} Q_{3}\right)-6\left(P_{3} P_{4}+Q_{3} Q_{4}\right)\right] \\
& \frac{\mathrm{d} Q_{2}}{\mathrm{~d} a_{3}}=-\frac{\Gamma K^{2}}{8}\left[-2 P_{1} Q_{1}+6\left(P_{1} Q_{3}-P_{3} Q_{1}\right)+16\left(P_{2} Q_{4}-P_{4} Q_{2}\right)\right] \\
& \frac{\mathrm{d} P_{2}}{\mathrm{~d} a_{3}}=\frac{\Gamma K^{2}}{8}\left[\left(P_{1}^{2}-Q_{1}^{2}\right)-6\left(P_{1} Q_{3}+P_{3} Q_{1}\right)-16\left(P_{2} Q_{4}+P_{4} Q_{2}\right)\right] \\
& \frac{\mathrm{d} Q_{3}}{\mathrm{~d} a_{3}}=-\frac{\Gamma K^{2}}{12}\left[-6\left(P_{1} Q_{2}+P_{2} Q_{1}\right)+12\left(P_{1} Q_{4}-P_{4} Q_{1}\right)\right] \\
& \frac{\mathrm{d} P_{3}}{\mathrm{~d} a_{3}}=\frac{\Gamma K^{2}}{12}\left[6\left(P_{1} P_{2}-Q_{1} Q_{2}\right)-12\left(P_{1} P_{4}+Q_{1} Q_{4}\right)\right] \\
& \frac{\mathrm{d} Q_{4}}{\mathrm{~d} a_{3}}=-\frac{\Gamma K^{2}}{16}\left[-16 P_{2} Q_{2}-12\left(P_{3} Q_{1}+P_{1} Q_{3}\right)\right] \\
& \frac{\mathrm{d} P_{4}}{\mathrm{~d} a_{3}}=\frac{\Gamma K^{2}}{16}\left[8\left(P_{2}^{2}-Q_{2}^{2}\right)+12\left(P_{3} P_{1}-Q_{3} Q_{1}\right)\right] .
\end{aligned}
$$

Pour simplifier les calculs, choisissons la phase du fondamental de façon que $P_{1}\left(a_{3}\right)=0$. Les équations précédentes montrent que :

$$
Q_{2}\left(a_{3}\right)=P_{2}\left(a_{3}\right)=Q_{4}\left(a_{3}\right)=0
$$

le déplacement $u_{3}$ peut donc s'écrire :

$$
\begin{aligned}
u_{3}=Q_{1}\left(a_{3}\right) \sin \left(K a_{3}\right. & -\Omega t)+P_{2}\left(a_{3}\right) \cos 2\left(K a_{3}-\Omega t\right) \\
& +Q_{3}\left(a_{3}\right) \sin 3\left(K a_{3}-\Omega t\right) \\
& +P_{4}\left(a_{3}\right) \cos 4\left(K a_{3}-\Omega t\right) .
\end{aligned}
$$

Cette relation montre que si la phase de référence est choisie de façon telle que le fondamental soit un sinus, tous les harmoniques pairs sont en cosinus et tous les harmoniques impairs sont en sinus. Cette propriété est une conséquence de l'absence de dispersion.

Nous avons supposé jusqu'ici que l'onde acoustique se propageait dans un milieu sans pertes. En désignant par $\alpha$ l'atténuation de l'onde à la fréquence fondamentale, l'atténuation de l'harmonique $n$ sera égale à $n^{2} \alpha$ puisque dans les isolants l'atténuation varie comme le carré de la fréquence. En posant :

$A=Q_{1}\left(a_{3}\right), \quad B=P_{2}\left(a_{3}\right), \quad C=\dot{Q}_{3}\left(a_{3}\right) \quad$ et $\quad D=P_{4}\left(a_{3}\right)$ nous obtenons finalement le système d'équations couplées :

$$
\frac{\mathrm{d} A}{\mathrm{~d} a_{3}}=-\alpha A-\frac{\Gamma K^{2}}{8}[-4 A B+12 B C-24 C D]
$$$$
\frac{\mathrm{d} B}{\mathrm{~d} a_{3}}=-4 \alpha B+\frac{\Gamma K^{2}}{8}\left[-A^{2}-6 A C-16 B D\right]
$$$$
\frac{\mathrm{d} C}{\mathrm{~d} a_{3}}=-9 \alpha C-\frac{\Gamma K^{2}}{8}[-4 A B-8 A D]
$$

$\frac{\mathrm{d} D}{\mathrm{~d} a_{3}}=-16 \alpha D+\frac{\Gamma K^{2}}{8}\left[4 B^{2}-6 A C\right]$.

Dans le cas général, ce système d'équations différentielles ne peut être résolu que par calcul numérique en imposant les conditions initiales (à $\left.a_{3}=0\right)$ :

$$
B(0)=C(0)=D(0)=0 .
$$

La valeur $A(0)$ est définie à partir de la puissance de l'onde acoustique fondamentale au niveau du transducteur par la relation :

$$
P_{\mathrm{ac}}=\frac{\rho v^{3} W \cdot e \cdot K^{2} A^{2}}{2}
$$


$W$ et $e$ étant respectivement la largeur et la hauteur du transducteur.

La technique de calcul sur ordinateur nous a permis d'obtenir directement les courbes de variation des puissances acoustiques aux différentes fréquences harmoniques en fonction de la distance parcourue dans le cristal en prenant comme paramètre la puissance acoustique fondamentale. La valeur de la constante $I$ peut être trouvée à l'aide d'une solution approchée valable au début de la propagation de l'onde dans le cristal et par des faibles puissances acoustiques.

C. Solution APPROCHÉE. - Considérons maintenant le début de la propagation du cristal dans le cas où l'onde fondamentale transporte une puissance relativement faible. Le transfert d'énergie entre l'onde fondamentale et les différents harmoniques reste alors très faible $(D \ll C \ll B \ll A)$ et dans le cas d'un milieu avec faibles pertes, nous pouvons négliger l'atténuation et supposer que l'amplitude de l'onde fondamentale reste constante. Avec ces hypothèses, nous obtenons en intégrant le système (16) :

$$
\begin{aligned}
& B \simeq-\frac{\Gamma K^{2}}{8} A^{2} a_{3} \\
& C \simeq-\frac{\Gamma^{2} K^{4}}{32} A^{3} a_{3}^{2} \\
& D \simeq \frac{\Gamma^{3} K^{4}}{96} A^{6} a_{3}^{3} .
\end{aligned}
$$

En exprimant la puissance acoustique de chaque harmonique en fonction de la puissance acoustique fondamentale à l'aide des relations (17) et (18), nous obtenons les propriétés suivantes qui permettront d'interpréter les résultats expérimentaux :

- la puissance du second harmonique est proportionnelle au carré de la distance parcourue dans le cristal, au carré de la puissance fondamentale et au carré de la fréquence fondamentale,

- la puissance du troisième harmonique est proportionnelle au cube de la puissance fondamentale et à la puissance quatrième de la fréquence fondamentale et de la distance parcourue dans le cristal,

- la puissance du quatrième harmonique est proportionnelle à la puissance quatrième de la puissance fondamentale et à la puissance sixième de la fréquence fondamentale et de la distance parcourue dans le cristal.

Ces résultats ne sont valables que pour des faibles puissances acoustiques et qu'au début de la propagation. Néanmoins, ils permettent de déterminer la valeur de la constante de couplage $\Gamma$. Nous montrerons en particulier qu'il est possible d'obtenir directement la valeur de $\Gamma$ à partir du rapport de la puissance acoustique du second harmonique et de la puissance acoustique fondamentale mesurées au même point dans le cristal et à faible niveau.
III. Etude expérimentale. - A. PRINCIPE DE LA MÉTHODE UTILISÉE. - Dans tout ce travail, nous avons utilisé la diffraction de la lumière dans le régime de Bragg par les ondes acoustiques [22] [23] afin d'étudier leur propagation en un point quelconque du cristal. Le principe de la méthode est décrit à la figure 1 .

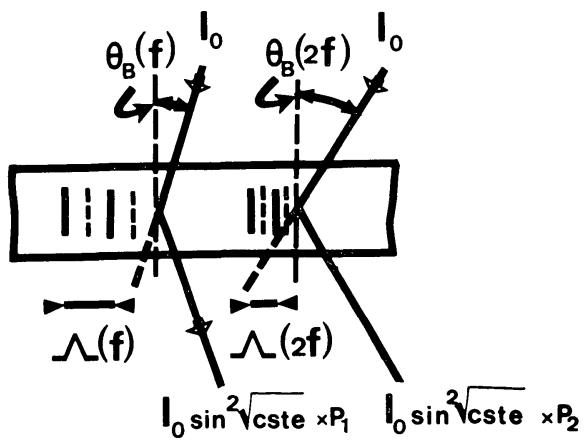

(a)

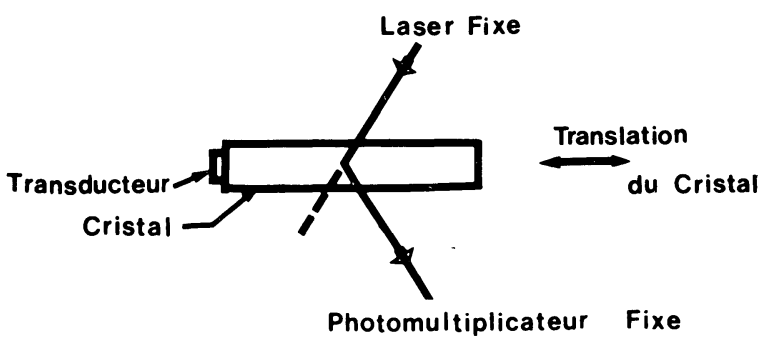

(b)

FIG. 1. - Principe de la méthode de détection des harmoniques ultrasonores par effet photo-élastique.

En faisant varier l'angle d'incidence du faisceau lumineux sur les plans d'onde acoustique, nous obtenons une analyse harmonique de la déformation qui se propage dans le cristal. En effet, si l'onde acoustique fondamentale de fréquence $f$ est détectée par un angle de Bragg $\theta_{\mathbf{B}}(f)$ défini par:

$$
\sin \theta_{\mathbf{B}}(f)=\frac{\lambda}{2 \Lambda}
$$

$\lambda$ étant la longueur de la lumière, $\Lambda$ la longueur d'onde acoustique, l'harmonique $n$ de l'onde fondamentale sera détectée pour un angle de Bragg $\theta_{\mathrm{B}}(n f)$ défini par :

$$
\sin \theta_{\mathrm{B}}(n f)=n \cdot \frac{\lambda}{2 \Lambda}=n \sin \theta_{\mathrm{B}}(f)
$$

et pour les angles petits :

$$
\theta_{\mathbf{B}}(n f) \simeq n \theta_{\mathbf{B}}(f) .
$$

En déplaçant le cristal parallèlement à l'axe de propagation de l'onde acoustique, nous pouvons mesurer la variation de la puissance de chaque harmonique en fonction de la distance de propagation. En effet, le 
rapport de l'intensité lumineuse diffractée $I_{\text {diffr }}$ par une onde acoustique de puissance $P_{\text {ac }}$ et de l'intensité lumineuse incidente $I_{0}$ est égal à : [24]

$$
\frac{I_{\mathrm{diff}}}{I_{0}}=\sin \sqrt[2]{\frac{k_{0}^{2}}{8} \frac{n^{6} p^{2}}{\rho v^{3}} \frac{W}{e} P_{\mathrm{ac}}}
$$

où $W$ et $e$ sont respectivement la largeur et la hauteur $\mathrm{du}$ faisceau acoustique, $k_{0}$ est le vecteur d'onde lumineux, $n$ l'indice optique du cristal, $p$ la constante photo-élastique mise en jeu dans l'interaction acoustooptique.

Cette relation montre que l'intensité lumineuse diffractée est directement reliée à la puissance acoustique de l'onde détectée et est indépendante de sa fréquence. La sonde optique utilisée permet donc de mesurer directement les puissances relatives des harmoniques de l'onde acoustique fondamentale en chaque point du cristal et évite en particulier le problème délicat du calibrage des transducteurs qui seraient utilisés comme récepteurs.

Cette méthode permet en outre de mesurer directement la constante de couplage $\Gamma$. En effet, pour de faibles puissances acoustiques, la relation (22) devient :

$$
\frac{I_{\mathrm{diff}}}{I_{0}}=\frac{k_{0}^{2}}{8} \cdot \frac{n^{6} p^{2}}{\rho v^{3}} \cdot \frac{W}{e} \cdot P_{\mathrm{ac}} .
$$

En remplaçant $P_{\text {ac }}$ par l'expression (17), nous obtenons l'intensité lumineuse diffractée par l'onde fondamentale :

$$
\frac{I_{1}}{I_{0}}=\frac{k_{0}^{2}}{8} \cdot\left(\frac{n^{6} p^{2}}{\rho v^{3}}\right) \cdot \frac{W}{e} \cdot \frac{\rho v^{3} W \cdot e K^{2} A^{2}}{2}
$$

et par le second harmonique :

$$
\frac{I_{2}}{I_{0}}=\frac{k_{0}^{2}}{8} \cdot\left(\frac{n^{6} p^{2}}{\rho v^{3}}\right) \cdot \frac{W}{e} \cdot \frac{\rho v^{3} W e 4 K^{2} B^{2}}{2}
$$

Les expressions (24a) et (24b) permettent de trouver le rapport $B / A^{2}$ :

$$
\frac{B}{A^{2}}=\left(\frac{I_{0} I_{2}}{I_{1}^{2}}\right)^{1 / 2} \cdot \frac{k_{0} n^{3} p W \cdot K}{8} .
$$

Ce rapport $B / A^{2}$ est relié à la constante de couplage $\Gamma$ par la relation (18a) qui peut s'écrire, en tenant compte de l'atténuation dans le cristal :

$$
\frac{B}{A^{2}}=-\frac{\Gamma}{8} K^{2} a_{3} \mathrm{e}^{-2 \alpha a_{3}}
$$

Nous obtenons, à partir de (25) et (26), la relation suivante :

$$
\Gamma=-\left(\frac{I_{0}}{I_{1}} \cdot \frac{I_{2}}{I_{1}}\right)^{1 / 2} \cdot \frac{k_{0} n^{3} p W}{K a_{3}} \cdot \mathrm{e}^{-2 \alpha a_{3}} .
$$

Cette relation montre que la constante de couplage $\Gamma$ peut être déterminée en mesurant les deux rapports $I_{1} / I_{0}$ et $I_{2} / I_{1}$ au même point $\left(a_{3}\right)$ du cristal et à faible niveau de puissance acoustique.
B. Méthodes de mesure. - Il est assez difficile de mesurer le coefficient $\Gamma$ à très faible niveau à cause de la très grande dynamique des signaux à détecter. Le problème est encore compliqué par le très grand coefficient de couplage opto-élastique de molybdate de plomb. Pour augmenter la précision des mesures, nous avons été amenés à utiliser plusieurs montages expérimentaux décrits dans ce paragraphe.

Il est d'abord nécessaire de contrôler soigneusement l'homogénéité du faisceau acoustique. Lorsque le couplage photo-élastique est faible, le champ électrique $\mathbf{E}_{1} \mathrm{du}$ faisceau optique diffracté est de la forme [24].

$$
\mathbf{E}_{1}=\mu \int_{-W / 2}^{W / 2} \frac{\Delta \varepsilon}{\varepsilon} \cdot \mathrm{e}^{\mathrm{i} \Delta K \cdot x} \mathrm{~d} x
$$

où $\mu$ est un facteur constant et $\Delta K$ une quantité proportionnelle à la différence entre l'angle de Bragg et l'angle d'incidence. Dans les conditions idéales d'interaction :

$$
\mathbf{E}_{1}=\mu \int_{-W / 2}^{W / 2} \frac{\Delta \varepsilon(x)}{\varepsilon} \cdot \mathrm{d} x
$$

Pour l'onde fondamentale, $\Delta \varepsilon(x) / \varepsilon$ est proportionnel à $\sqrt{P(x)}$ où $P(x)$ est la densité de puissance acoustique :

$$
E_{11}=h_{1} \int_{-W / 2}^{W / 2} \sqrt{P(x)} \cdot \mathrm{d} x .
$$

Pour la seconde harmonique, $\Delta \varepsilon(x) / \varepsilon$ n'est plus proportionnel à $\sqrt{P(x)}$ mais à $f(\sqrt{P(x)})$ où $f$ est une fonction voisine de la fonction $u-0 \rightarrow u^{2}$ et le champ électrique du faisceau optique diffracté est de la forme :

$$
E_{12}=h_{2} \int_{-W / 2}^{W / 2} f(\sqrt{P(x)}) \cdot \mathrm{d} x .
$$

Pour que les formules (22) et (26) soient valables, il est donc nécessaire que $\sqrt{P(x)}$ soit une constante ; dans le cas contraire, il serait nécessaire de faire intervenir la fonction $f$ qu'on ne peut connaître a priori.

Le contrôle de l'homogénéité du faisceau acoustique pour des rendements opto-élastiques inférieurs à $10 \%$ ne pose pas de problèmes, il suffit en effet de vérifier que l'intensité diffractée en fonction de l'angle d'incidence est de la forme [24] :

$$
\frac{\sin ^{2} Y}{Y^{2}}
$$

où $Y$ est proportionnel à $\Delta \theta$.

Pour des rendements élevés (puissances acoustiques supérieures à $100 \mathrm{~mW}$ ), le contrôle de l'homogénéité pose des problèmes relativement difficiles à résoudre. La première méthode consiste à coller en bout du cristal de molybdate de plomb un matériau comme la silice ayant un couplage très faible et de vérifier que le diagramme de diffraction reste de la forme (31) quel que soit le niveau acoustique. Nous avons abandonné cette méthode car il est assez difficile d'obtenir 
un collage suffisamment homogène pour faire un contrôle efficace.

Il a été montré [24] que le rendement de l'interaction opto-acoustique en fonction de la puissance acoustique et de l'angle d'incidence est de la forme :

$$
\mathcal{R}=\frac{X^{2}}{X^{2}+Y^{2}} \cdot \sin ^{2} \sqrt{X^{2}+Y^{2}}
$$

où $X$ est fonction de $\sqrt{P}$ et $Y$ proportionnel à $\Delta \theta$.

On estime que le faisceau acoustique est homogène si le résultat expérimental est suffisamment voisin de (32).

Le montage expérimental (Fig. 2) se compose d'un laser hélium-néon de $5 \mathrm{~mW}$ de puissance. Le faisceau

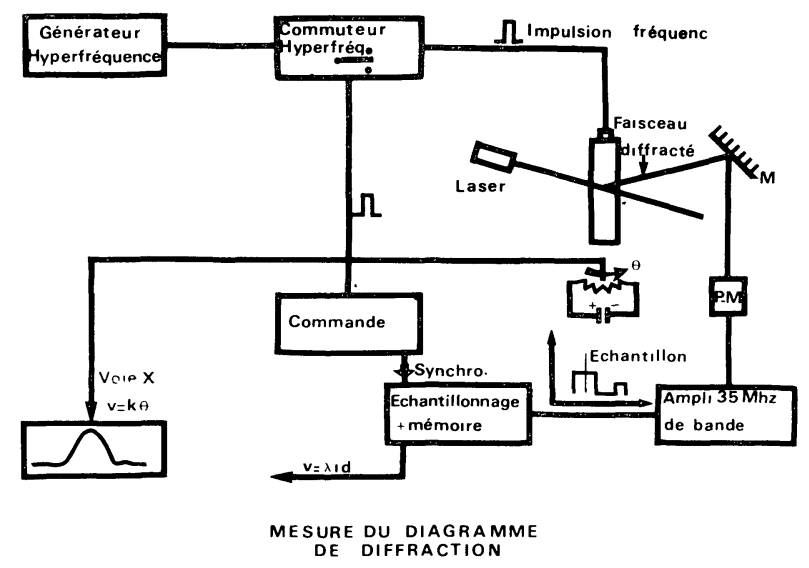

FIG. 2. - Schéma du dispositif de relevé des diagrammes de diffraction des faisceaux acoustiques.

optique est focalisé sur le faisceau acoustique par une lentille cylindrique de $20 \mathrm{~cm}$ de focale. Le faisceau optique dévié est repris par une lentille cylindrique symétrique de la précédente, puis focalisé par une lentille sphérique sur une photodiode. Le cristal est porté par une platine tournante dont la rotation est couplée à un potentiomètre délivrant une tension proportionnelle à l'angle de rotation. Le transducteur est alimenté par un générateur hyperfréquence modulé en impulsions pour éviter d'appliquer des puissances moyennes trop importantes et pour éviter toute erreur systématique due à des signaux parasites lumineux continus. Le signal fourni par la photodiode est amplifié par un amplificateur à large bande. Après échantillonnage, mise en mémoire et filtrage, le signal est appliqué à la voie $X$ d'un enregistreur, la tension fournie par le potentiomètre étant appliquée à la voie $Y$.

La linéarité de la chaîne de mesure est contrôlée en vérifiant que le rendement est proportionnel à la puissance électrique appliquée au transducteur pour des rendements inférieurs à $10 \%$. Le faisceau lumineux est ensuite atténué de $10 \mathrm{~dB}$.

Le relevé du niveau diffracté en fonction de la distance pour les rendements supérieurs à $1 \%$ se fait au moyen du montage décrit plus haut dans lequel on remplace les lentilles cylindriques par des lentilles sphériques pour concentrer le faisceau en un point précis du cristal. Un déplacement calibré du cristal devant le faisceau laser permet de faire varier la distance entre la zone d'interaction et le transducteur. La chaîne de mesure est identique à la précédente. La porte de l'échantillonneur est déplacée manuellement dans le temps de manière à échantillonner le signal fourni par la photodiode au même endroit et éliminer ainsi toute erreur due à une impulsion hyperfréquence imparfaite. Cette méthode est précise et relativement sensible car elle permet un filtrage efficace après l'échantillonnage. Elle a le défaut d'être lente et par conséquent sensible aux instabilités des chaînes d'amplification ou du niveau de sortie du générateur hyperfréquence.

Pour des niveaux acoustiques tels que le rendement soit supérieur à $10 \%$, nous avons modifié le montage précédent pour rendre l'enregistrement plus rapide. Le faisceau optique est élargi au moyen d'un système anamorphaseur à prismes [25] permettant d'obtenir un faisceau optique absolument parallèle. Le transducteur est alimenté par une impulsion très brève, d'une durée de l'ordre de $100 \mathrm{~ns}$. La photodiode reçoit donc dans le temps un signal dépendant du niveau acoustique au point d'abscisse $a_{3}=v\left(t-t_{0}\right)$. Le signal fourni par la photodiode est stroboscopé par un oscilloscope à échantillonnage et enregistré au moyen d'un enregistreur $X-Y$.

Cette méthode très rapide mais peu sensible s'est révélée extrêmement précise pour le relevé du niveau des harmoniques 2,3 et 4 pour des puissances acoustiques supérieures à $1 \mathrm{~W}$.

Lorsque le rendement est inférieur à $10 \%$, il est cependant préférable d'utiliser un montage optique superhétérodyne sensible non plus à l'intensité lumineuse diffractée mais à l'amplitude de l'onde acoustique. Il est ainsi possible de détecter des densités de puissance acoustique inférieures au nanowatt par millimètre carré.

La pulsation du champ électrique $\mathbf{E}_{1}$ du faisceau optique dévié est égale à :

$$
\omega_{1}=\omega_{0}+\Omega
$$

où $\omega_{0}$ est la pulsation du champ électrique du faisceau optique incident et $\Omega$ la pulsation de l'onde acoustique.

D'après l'expression (28), dans les conditions de Bragg $(\Delta K=0)$ et pour un transducteur homogène, le champ diffracté $\mathbf{E}_{1}$ est de la forme :

$$
\mathbf{E}_{1}=h \cdot \frac{\Delta \varepsilon}{\varepsilon} \cdot \mathrm{e}^{\mathrm{i}\left[\left(\omega_{0}+\Omega\right) t+\varphi\right]} \mathbf{e}_{0}
$$

$\varphi$ étant un terme de phase qui dépend du point d'interaction :

$$
\varphi=-\left(\mathbf{k}_{0}+\mathbf{K}\right) \cdot \mathbf{r} .
$$

Si l'on fait battre le rayon lumineux de champ électrique $\mathbf{E}_{1}$ avec un rayon lumineux de vecteur électrique $\mathbf{E}_{0}$ :

$$
\mathbf{E}_{0}=\mathbf{e}_{0} \cdot \mathrm{e}^{\mathrm{i}\left(\omega_{0} t-\mathbf{k}_{0} \cdot \mathbf{r}+\varphi^{\prime}\right)}
$$


le champ électrique résultant est :

$\mathbf{E}=\mathbf{e}_{0}\left[\mathrm{e}^{\mathrm{i}\left(\omega_{0} t-\mathbf{k}_{0} \cdot \mathbf{r}+\varphi^{\prime}\right)}\right] \cdot\left[1+h \frac{\Delta \varepsilon}{\varepsilon} \mathrm{e}^{\mathrm{i}\left(\Omega t-\mathbf{K} \cdot \mathbf{r}-\varphi^{\prime}\right)}\right]$.

Le courant de la photodiode est proportionnel au carré du module de $\mathbf{E}$ :

$$
\begin{aligned}
I= & \left|\mathbf{e}_{0}\right|^{2} \times \\
& \times\left[1+\left(h \frac{\Delta \varepsilon}{\varepsilon}\right)^{2}+2 h \frac{\Delta \varepsilon}{\varepsilon} \cos \left(\Omega t-\mathbf{K} \cdot \mathbf{r}-\varphi^{\prime}\right)\right] .
\end{aligned}
$$

Les deux premiers termes sont constants dans le temps et génèrent un courant constant dans la photodiode. Ce courant est écoulé à la masse à travers une selfinductance. Le courant alternatif est appliqué à un récepteur ayant une impédance d'entrée de $50 \Omega$. La tension $V$ apparaissant aux bornes de la photodiode est donc :

$$
V=50 \cdot\left|\mathbf{e}_{0}\right|^{2} \cdot 2 h \frac{\Delta \varepsilon}{\varepsilon} \cos (\Omega t-\mathbf{K} \cdot \mathbf{r})
$$

si on omet le terme de phase constant $\varphi^{\prime}$ fixé par le montage.

Si le cristal est déplacé dans une direction parallèle à $\mathbf{K}$, la fonction $V$ est périodique en $a_{3}$ et de période égale à celle de l'onde acoustique. Il est donc possible, en déplaçant le cristal devant le faisceau lumineux, d'enregistrer les variations de l'indice du cristal tout au long du parcours de l'onde ultrasonore.

Pour plus de commodité, nous avons cherché à enregistrer directement l'enveloppe du signal $V$ en le faisant battre avec un signal hyperfréquence de référence de pulsation $\Omega$ :

$$
V_{1}=v_{1} \mathrm{e}^{\mathrm{i} \Omega t} .
$$

Le signal hyperfréquence résultant est de la forme :

$$
v_{\mathrm{s}}=v_{1} \mathrm{e}^{\mathrm{i} \Omega t}+2\left|\mathbf{e}_{0}\right|^{2} h \frac{\Delta \varepsilon}{\varepsilon} \mathrm{e}^{\mathrm{i}\left(\Omega t-K a_{3}\right)}
$$

dont l'enveloppe a pour équation :

$$
\sqrt{v_{1}^{2}+4\left(\left|\mathbf{e}_{0}\right|^{2} h \frac{\Delta \varepsilon}{\varepsilon}\right)^{2}+4 v_{1}\left|\mathbf{e}_{0}\right|^{2} \cdot h \frac{\Delta \varepsilon}{\varepsilon} \cos K a_{z}} .
$$

Le potentiel constant $v_{1}$ sert à polariser la diode de détection à une valeur fixe (le deuxième terme étant faible par rapport à $v_{1}^{2}$ ).

Le potentiel redressé est alors strictement proportionnel à :

$$
\left|\mathbf{e}_{0}\right| \cdot h \cdot \frac{\Delta \varepsilon}{\varepsilon} \cdot \cos K \cdot a_{3}
$$

Il suffit d'enregistrer ce signal en fonction de $a_{3}$ pour visualiser strictement les variations d'indice à l'intérieur du cristal. Cette méthode est remarquable par sa sensibilité : elle permet de mettre en évidence des puissances acoustiques de l'ordre du nanowatt.
C. RÉAlisation eXPéRIMENTAle. - 1. Partie optique. - La réalisation expérimentale peut paraître délicate. Cependant, depuis plusieurs années nous avons travaillé sur ce genre de problème pour réaliser des lignes à retard continûment variable [26]. La meilleure structure optique que nous ayons mise au point est représentée à la figure 3 . Le faisceau sortant

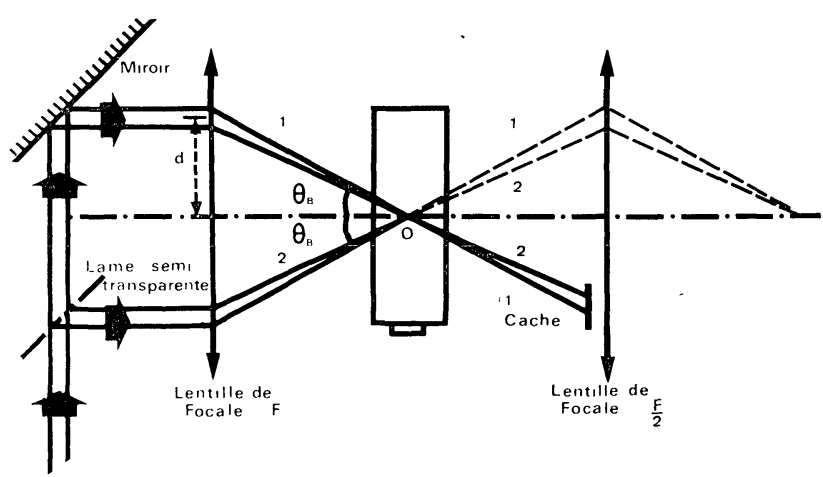

FIG. 3. - Schéma optique du dispositif hétérodyne de détection des ultrasons.

du laser est divisé en deux faisceaux identiques au moyen d'une lame semi-transparente et d'un miroir. Ces deux faisceaux sont concentrés par une lentille de focale $F$ appropriée sur le cristal. La distance $2 d$ des faisceaux est telle que $d / F=\theta_{\mathrm{B}}$. D'autre part, Kogelnik [27] a montré que l'enveloppe d'un faisceau optique ayant une distribution gaussienne est un hyperboloïde de révolution dont le cône asymptote a pour angle au sommet au foyer de la lentille :

$$
\psi=\frac{8}{\pi} \frac{\lambda}{\Phi}
$$

et dont le diamètre du cercle à l'étranglement est $\Phi_{0}$ :

$$
\Phi_{0}=\frac{8}{\pi} \frac{\lambda}{\Phi} F
$$

où $\Phi$ est le diamètre du faisceau optique sortant $\mathrm{du}$ laser (Fig. 4). Ainsi le faisceau optique reste parallèle sur une distance $D$ qui est supérieure à la largeur $W$ du faisceau ultrasonore si la focale $F$ est suffisamment grande.

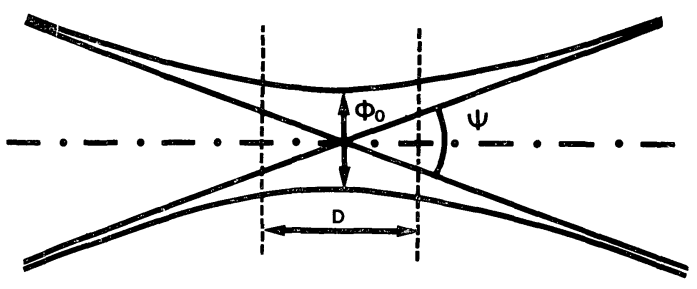

FIG. 4. - Forme d'un faisceau optique gaussien au foyer d'une lentille convergente.

Ce montage permet d'obtenir de grandes variations de fréquence (nous avons réalisé un déflecteur à 1,5 $\mathrm{GHz}$ avec une bande passante de $600 \mathrm{MHz}$ ) [28]. 
Cela signifie aussi que le réglage est assez peu critique. Lorsque le couplage est grand, le faisceau (2) est perturbé et la théorie précédente doit être corrigée en remplaçant dans la formule (38) le facteur $\left|\mathbf{e}_{0}\right|^{2}$ $\operatorname{par}\left|\mathbf{e}_{0}\right|^{2} \cdot\left(1-\mathcal{R}^{2}\right)$.

La dernière lentille de focale $F / 2$ concentre les faisceaux optiques sur la photodiode dont la surface sensible est très petite pour obtenir des bandes passantes importantes. En effet, la capacité de la photodiode étant proportionnelle à sa surface, la bande passante est inversement proportionnelle à la surface. Pour cette raison, nous avons utilisé la photodiode HP 7201 de Hewlett Packard. Le trajet des rayons lumineux étant symétrique par rapport à la lentille et le point $O$ étant fixe, les rayons lumineux arrivent sur la photodiode quelle que soit la fréquence acoustique. Ce montage permet une plus grande facilité de réglage.

2. Partie hyperfréquence (Fig. 5). - Elle comprend un oscillateur hyperfréquence qui délivre un signal

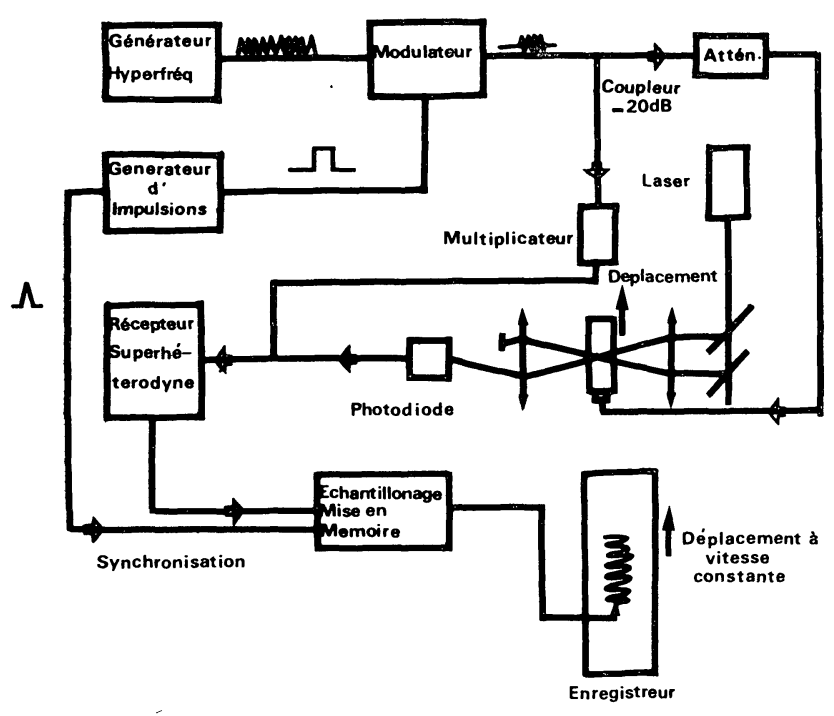

FIG. 5. - Schéma optique et électronique du relevé hétérodyne des niveaux acoustiques.

modulé en impulsions pour éviter des phénomènes thermiques parasites. Après amplification, une partie de ce signal est prélevée et atténuée de manière à constituer le signal de référence. La fréquence de ce signal de référence est multipliée par le rang de l'harmonique que l'on désire détecter. L'autre partie du signal est appliquée au transducteur par l'intermédiaire d'un atténuateur permettant de faire varier la puissance.

Le signal de la photodiode est appliqué après mélange avec le signal de référence à un récepteur superhétérodyne de bande passante égale à $4 \mathrm{MHz}$ (suffisante pour amplifier les impulsions sans trop les déformer). Après détection, le signal est échantillonné et mis en mémoire entre deux impulsions successives. On dispose alors d'un signal continu qui peut être enregistré.
3. La ligne de diffraction. - Elle est constituée d'un barreau de molybdate de plomb de $6 \times 6 \times 15 \mathrm{~mm}$ sur lequel a été soudé un transducteur de niobate de lithium. Les principales caractéristiques physiques de molybdate de plomb sont les suivantes [10]:

$$
\begin{aligned}
\text { - vitesse acoustique } v & =3632 \mathrm{~m} / \mathrm{s} \\
\text { - indice de réfraction } n & =2,386 \\
\text { - masse volumique } \rho & =6,950 \times 10^{3} \mathrm{~kg} / \mathrm{m}^{3}
\end{aligned}
$$

- facteur de mérite opto-acoustique : 23,7 fois celui de la silice (pour les ondes acoustiques longitudinales se propageant suivant $(001)-$ ).

Le transducteur a une surface utile de $(0,7 \times 4,2) \mathrm{mm}^{2}$ et a une bande passante comprise entre 150 et $280 \mathrm{MHz}$. Son impédance électrique de quelques ohms a été adaptée à $80 \Omega$ avec un taux d'ondes stationnaires inférieur à 2 (pour éviter la réflexion des ondes hyperfréquences sur le générateur). Avec une telle adaptation, la perte de conversion électromécanique du transducteur est environ $4 \mathrm{~dB}$.

D. Résultats expérimentaux. - Nous avons d'abord contrôlé l'homogénéité des faisceaux acoustiques. Les figures 6 et 7 représentent le rendement de

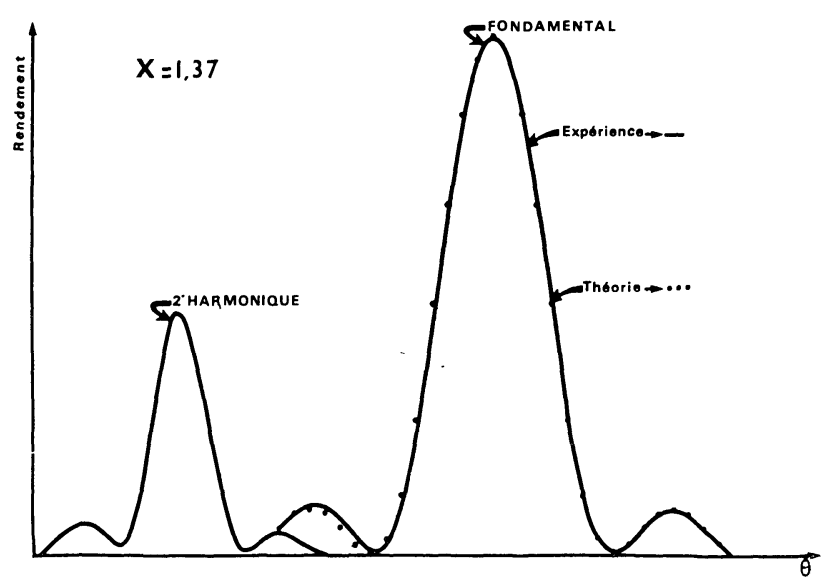

FIG. 6. - Diagramme de rayonnement à l'infini du faisceau acoustique fondamental et du $2^{\mathrm{e}}$ harmonique.

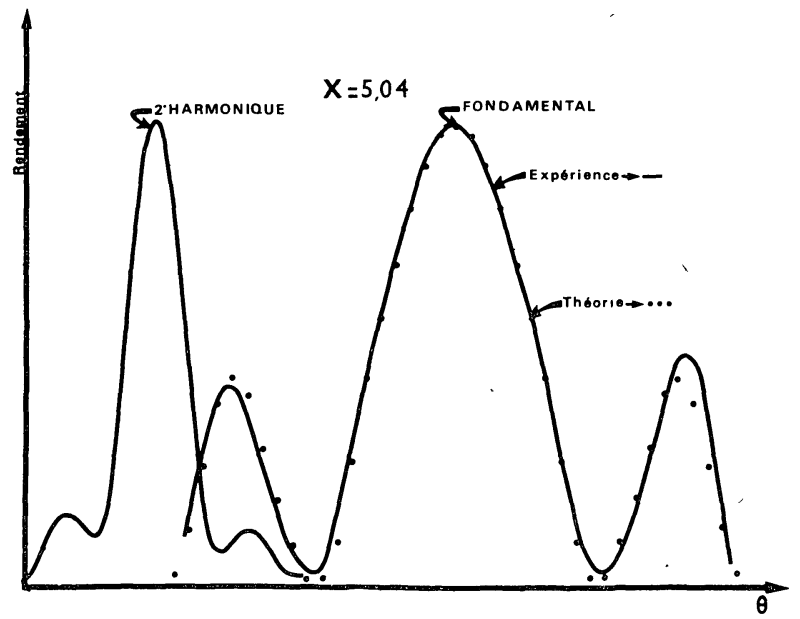

FIG. 7. - Diagramme de rayonnement à l'infini du faisceau acoustique pour $X=5,04$. 
l'interaction acousto-optique en fonction de l'angle d'incidence pour deux valeurs de $X$ correspondant à des puissances acoustiques respectivement égales à $780 \mathrm{~mW}$ et $11 \mathrm{~W}$. Nous avons en fait tracé toutes les courbes pour $0,5<X<6$ ( $X$ étant défini en (32)) L'accord entre la théorie et l'expérience montre que le

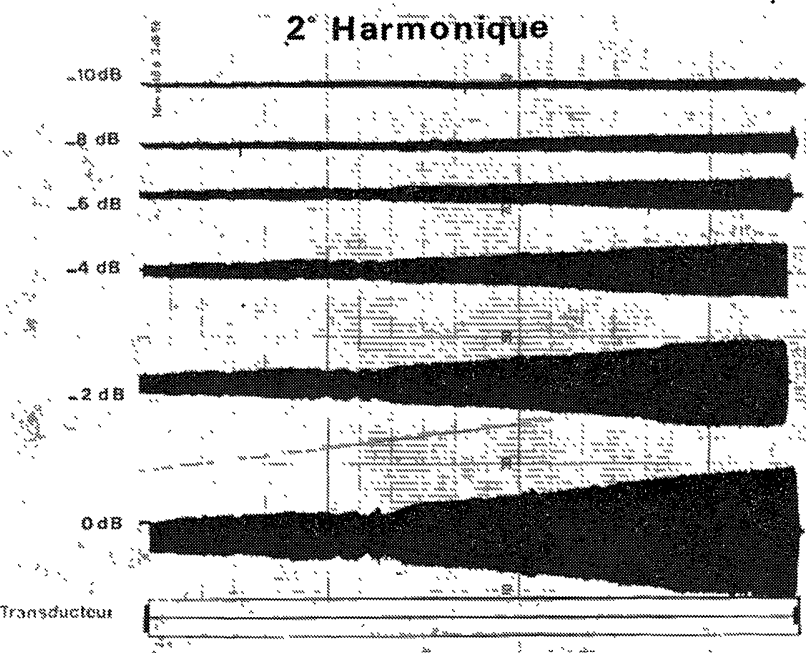

FIG. 8. - Enregistrement du niveau du $2^{\mathrm{e}}$ harmonique en fonction de la distance (Méthode hétérodyne).

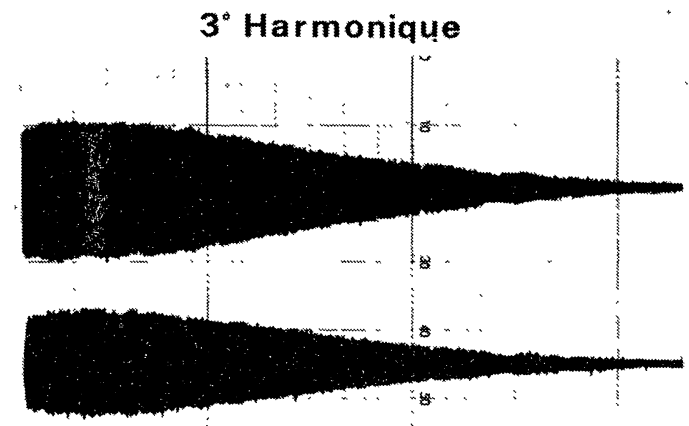

$.1 d B$

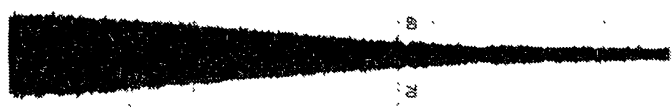

OdB

$.1 \mathrm{~dB}$

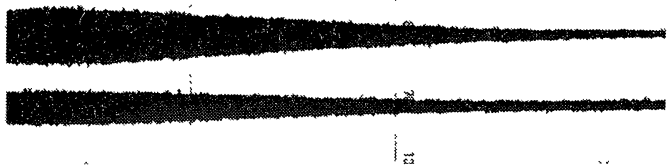

FIG. 9. - Enregistrement du niveau du $3^{\mathrm{e}}$ harmonique en fonction de la distance (Méthode hétérodyne).

transducteur a un rayonnement homogène pour toutes les puissances acoustiques. Les diagrammes de diffraction du second harmonique montrent que sa puissance est aussi quasi homogène. On peut donc estimer que les erreurs commises sur le calcul de $\Gamma$ dues aux inhomogénéités du transducteur sont négligeables.

Pour mesurer avec précision le coefficient $\Gamma$ il faut en fait combiner plusieurs méthodes de mesure. Il est

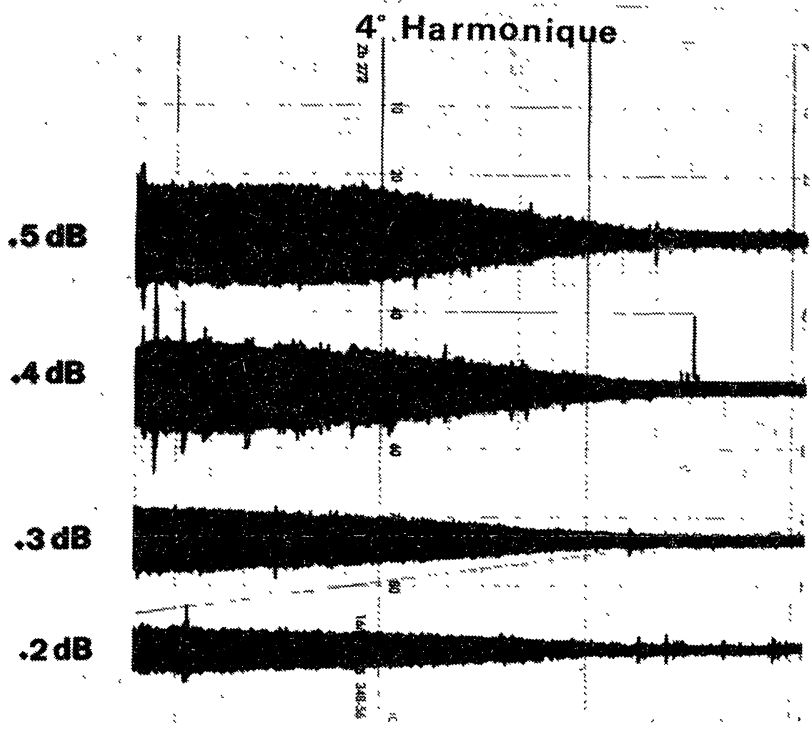

FIG. 10. - Enregistrement du niveau du $4 \mathrm{e}^{\mathrm{e}}$ harmonique en fonction de la distance (Méthode hétérodyne).

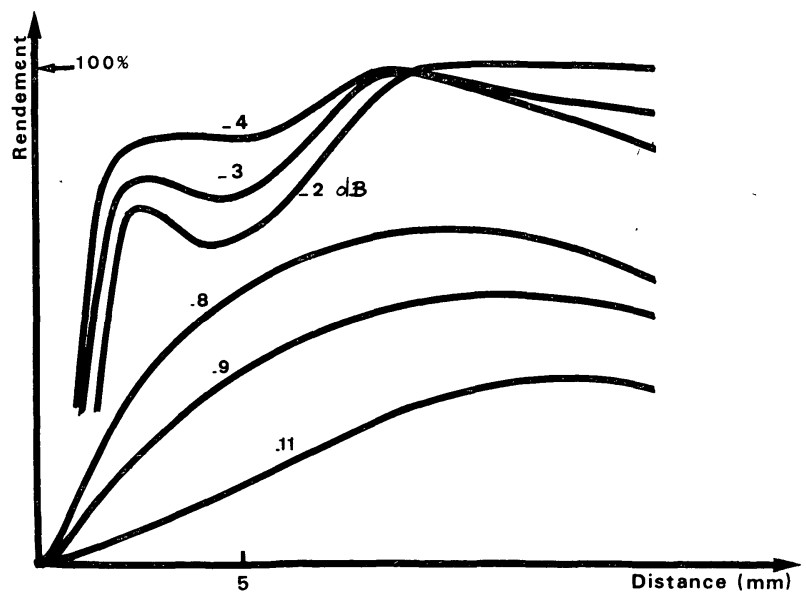

Fig. 11. - Enregistrement du niveau du $2^{\mathrm{e}}$ harmonique en fonction de la distance et de la puissance acoustique fondamentale.

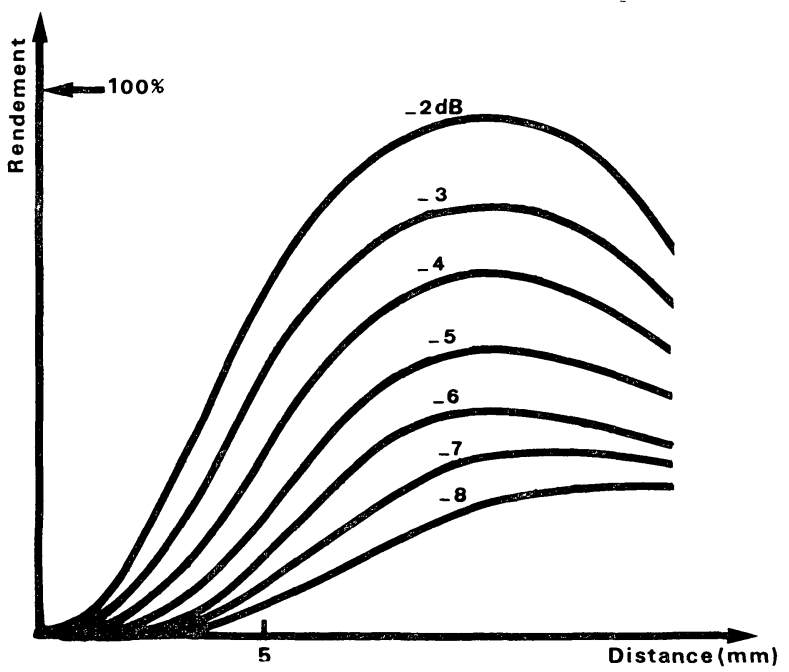

Fig. 12. - Enregistrement du niveau du $3^{\text {e harmonique en }}$ fonction de la distance et de la puissance acoustique fondamentale. 
en effet difficile de faire des mesures absolues avec un montage optique superhétérodyne. En prenant beaucoup de précautions et en utilisant un matériel hyperfréquence très perfectionné, il est à la rigueur possible d'obtenir les niveaux absolus avec une erreur de l'ordre de $\pm 1 \mathrm{~dB}$. Nous avons préféré utiliser la combinaison des trois méthodes exposées précédemment. Le montage superhétérodyne reste valable jusqu'à des rendements de l'ordre de $10 \%$. Il est alors possible d'utiliser la méthode de relevé point par point pour rétablir un niveau absolu correspondant à un point de mesure effectué au moyen du montage superhétérodyne.

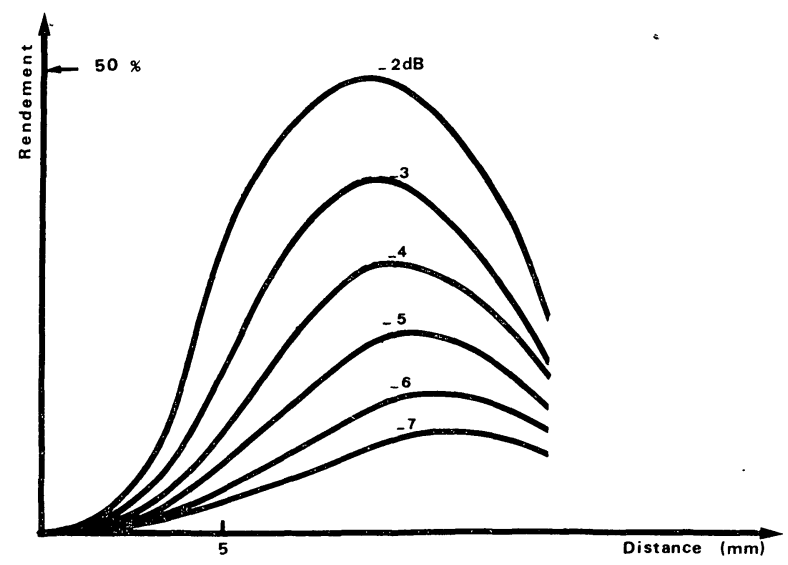

FIG. 13. - Enregistrement du niveau du $4^{\mathrm{e}}$ harmonique en fonction de la distance et de la puissance.

Les figures 8, 9, 10 représentent le relevé de l'amplitude des harmoniques au moyen de la méthode superhétérodyne en fonction de la distance entre le point d'interaction et le transducteur.

Les figures 11, 12, 13 représentent le relevé du niveau diffracté par les harmoniques à très haut niveau de puissance acoustique fondamentale, à l'aide de la seconde méthode.

IV. Comparaison entre résultats théoriques et résultats expérimentaux. - Nous avons déterminé la valeur de la constante de couplage $\Gamma$ en appliquant la méthode exposée au paragraphe III. A et à partir des résultats expérimentaux précédents. Pour une puissance acoustique fondamentale de $20 \mathrm{~mW}$, à la fréquence égale à $264 \mathrm{MHz}$, nous avons obtenu à la distance $a_{3}=14 \mathrm{~mm}$ :

$$
\frac{I_{1}}{I_{0}}=0,05, \quad \frac{I_{2}}{I_{1}}=0,02
$$

En appliquant la formule (27), nous avons obtenu :

$$
\Gamma=3+\frac{C_{333}}{C_{33}} \simeq-18
$$

Nous avons ensuite résolu le système d'équations couplées (16) avec les mêmes conditions initiales. Le meilleur accord entre la théorie et l'expérience a été trouvé pour $\Gamma=-17,5 \pm 1$. Les figures $14 a, b, c, d$ représentent les résultats obtenus à $264 \mathrm{MHz}$ pour différentes puissances acoustiques fondamentales $(20 \mathrm{~mW}, 100 \mathrm{~mW}, 500 \mathrm{~mW}, 1 \mathrm{~W})$. Les résultats ainsi obtenus nous permettent de mettre en évidence des phénomènes intéressants qui n'apparaissent pas au début de la propagation :

- lorsque le niveau de l'onde fondamentale augmente, l'onde fondamentale subit un excès d'atténuation dû au couplage anharmonique. L'atténuation des harmoniques (après passage du maximum) dépend de l'amplitude fondamentale initiale ;

- l'abscisse des maxima atteints par les amplitudes des harmoniques diminue lorsque l'amplitude fondamentale initiale augmente. Cette variation semble être due à la décroissance de plus en plus rapide de l'amplitude fondamentale.

L'étude théorique faite au paragraphe II en développant l'énergie libre du cristal jusqu'au $3^{\mathrm{e}}$ ordre et en ne tenant compte que des quatre premiers harmoniques a permis de prévoir les résultats expérimentaux et l'accord entre la théorie et l'expérience est pratiquement parfait, aux erreurs expérimentales près, pour les faibles puissances acoustiques $\left(P_{\mathrm{ac}} \leqslant 100 \mathrm{~mW}\right)$. Pour des puissances acoustiques de l'ordre du watt, le léger désaccord entre les résultats théoriques et expérimentaux montre que nos hypothèses deviennent moins valables. Pour obtenir de meilleurs résultats théoriques, il faudrait développer l'énergie libre du cristal à un ordre supérieur au troisième et tenir compte des harmoniques d'ordre supérieur à quatre, ce qui rendrait les calculs extrêmement compliqués.

V. Conclusion. - L'ensemble des résultats obtenus dans ce travail montre que la génération harmonique acoustique peut être interprétée en généralisant la loi de Hooke et en introduisant les constantes élastiques du troisième ordre. Les méthodes de mesure mises au point au cours de cette étude, en particulier la détection hétérodyne optique, ont permis de visualiser directement les effets non linéaires et d'évaluer la constante de couplage $\Gamma$ du molybdate de plomb :

$$
|\Gamma|=\left|3+\frac{C_{333}}{C_{33}}\right|=17,5 \pm 1
$$

que l'on peut comparer à celle du niobate de lithium par exemple [29]: $|\Gamma|=2,6$. Ces résultats peuvent intéresser à la fois les physiciens de l'état solide qui ont une information sur la constante élastique du $3^{\mathrm{e}}$ ordre $C_{333}$ du $\mathrm{PbMoO}_{4}$ et les nombreux utilisateurs des déflecteurs en $\mathrm{PbMoO}_{4}$ qui ont un ordre de grandeur des effets non linéaires non négligeables à haut niveau de puissance acoustique. 

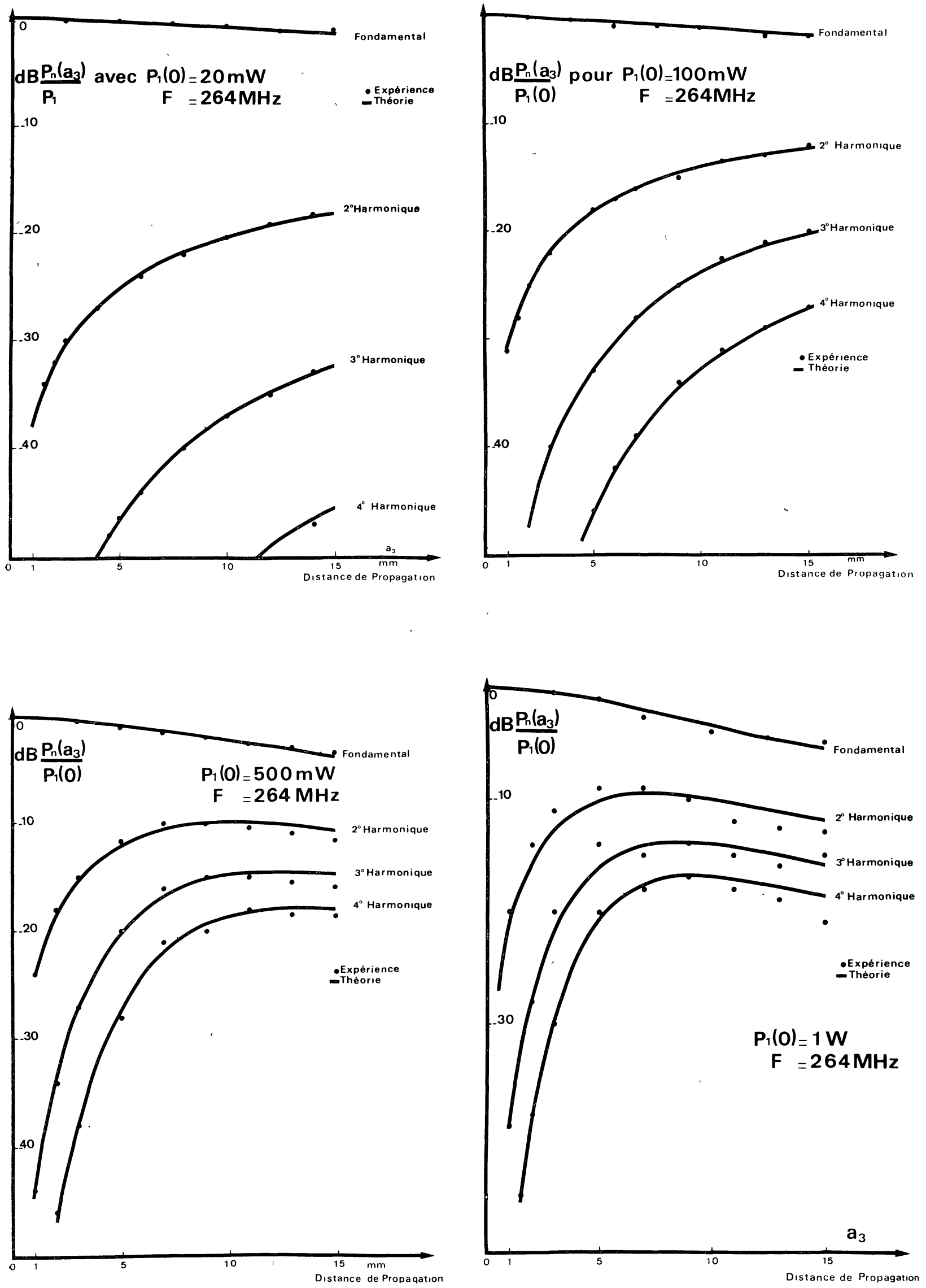

FIG. $14 a, b, c, d$. - Variation en fonction de la distance de la puissance des harmoniques 2, 3, 4 d'une onde fondamentale de puissance $-20 \mathrm{~mW}(a), 100 \mathrm{~mW}(b), 500 \mathrm{~mW}(c)$ et $1 \mathrm{~W}(d)$. 


\section{Bibliographie}

[1] Gordon (E. L.), Proc. IEEE, 1966, 54, 1391.

[2] Dixon (R. W.) et Gordon (E. I.), Bell Sys. Tech. J., 1967, 46, 367.

[3] Korpel (A.), Adler (R.), Desmares (P.) et WatSON (W.), Proc. IEEE, 1966, 54, 1429.

[4] Schulz (M. B.), Holland (M. G.) et Davis (L.), Appl. Phys. Lett., 1967, 11, 237.

[5] Collins (J. H.), Lean (E. G. H.) et Shaw (H. J.), Appl. Phys. Lett., 1967, 11, 240.

[6] Dixon (R. W.), J. Appl. Phys., 1967, 38, 5149.

[7] SмIth (T. M.) et Korpel (A.), IEEE J. Quantum Electron, QE-1, 283.

[8] Reintzes (J.) et Schulz (M. B.), J. Appl. Phys., 1968, $39,5254$.

[9] PINNOW (D. A.), IEEE J. Quantum Electron, 1970, QE-6, 223.

[10] Coquin (G. A.), Pinnow (D. A.) et Warner (A. W.), J. Appl. Phys., 1971, 42, 2162.

[11] Pinnow (D. A.) et Dixon (R. W.), Appl. Phys. Lett., $1968,13,156$

[12] Gedroits (A. A.) et Krasil'nikov (Y. A.), Sov. Phys. JETP, 1968, 16, 1122.

[13] CARr (P. H.), IEEE Trans. Sonics Ultrasonics, 1966, $13,103$.

[14] Parker (J. H.), Kelly (E. F.) et Bolef (D. I.), Appl. Phys. Lett., 1964, 5, 7.
[15] Rollins (F. R.), Appl. Phys. Lett., 1963, 2, 147.

[16] Breazeale (M. A.) et Thompson (D. O.), Appl. Phys. Lett., 1963, 3, 77.

[17] Buck (O.) et THOMPson (D. O.), Mater Sci. Eng., 1966 $1,117$.

[18] Richardson (B. A.), THOMpson (R. B.) et Wilkinson (C. D.W.), J. Acoust. Soc. Amer., 1968, 44, 1608.

[19] Thurston (R. N.), Physical Acoustics, Academic Press, New York, 1964, 1-A, 1.

[20] Thurston (R. N.) et Shapiro (M. J.), J. Acoust. Soc. Amer., 1967, 41, 111.

[21] Brugger (K.), J. Appl. Phys., 1965, 36, 759.

[22] Quate (C. F.), Wilkinson (C. D. W.) et Winslow (D. K.), Proc. IEEE, 1965, 53, 1604.

[23] Cohen (M. G.) et Gordon (E. I.), Bell System Tech. J., 1965, 44, 693.

[24] Torguet (R. J.), A publier.

[25] Gires (F.), Rev. Phys. Ap., 1969, 4, 505.

[26] ToRguet (R. J.), Rapport final Marché DRME 68, 34, 394, 00, 480, 7501.

[27] Kogelnik (H.), Appl. Opt., 1966, 10, 1550.

[28] Defranould (Ph.) et Torguet (R.), Ultrasonics Symposium, 1971, Miami, 0-7.

[29] McMahon (D. H.), J. Acoust. Soc. Amer.. 1968, 44, 1007. 